\title{
An expanding hadronic supercritical model for $\gamma$-ray burst emission
}

\author{
Ioulia Florou, ${ }^{a, *}$ Apostolos Mastichiadis ${ }^{a}$ and Maria Petropoulou ${ }^{a}$ \\ ${ }^{a}$ National \& Kapodistrian University of Athens, \\ Department of Physics, University Campus Zografos, GR 15783, Greece \\ E-mail: iflorou@phys.uoa.gr, amastich@phys.uoa.gr, mpetropo@phys.uoa.gr
}

Relativistic hadronic plasmas have an intriguing property, coined as hadronic supercriticality, according to which they can abruptly and efficiently release the energy stored in protons through photon outbursts. These photon flares may have a direct analogy to those observed from compact astrophysical objects, such as Gamma Ray Bursts (GRBs). Here, we investigate for the first time the manifestation and properties of hadronic supercriticality in adiabatically expanding sources. We consider the injection of relativistic protons in an expanding spherical volume with a radially decaying magnetic field and seek the parameters (e.g., proton injection luminosity) that drive the system to supercriticality. We then perform a Monte Carlo simulation for selecting the parameters of expanding, supercritical blobs and calculate the time-dependent electromagnetic signal from each one of them. We compute the $\gamma$-ray light curves and broadband photon spectra from the superposition of these blobs and present a physical picture for the prompt emission of a typical long GRB. We also provide the all-flavour neutrino fluxes expected in this model and compare them with those from the standard internal-shock scenario for GRBs.

$37^{\text {th }}$ International Cosmic Ray Conference (ICRC 2021)

July 12th - 23rd, 2021

Online - Berlin, Germany

\footnotetext{
${ }^{*}$ Presenter
} 


\section{Introduction}

The prompt emission of Gamma-Ray Bursts (GRBs) is usually characterised by rapid, highly variable light curves and non-thermal spectra having a peak at approximately $1 \mathrm{MeV}$. These observations imply the presence of ultra-relativistic outflows with bulk Lorentz factors greater than 100 and of very compact sources with strong magnetic fields. However, the origin of the prompt emission is not yet well understood. A possible candidate for the production of the $\gamma$-rays are the hadronic models, which also shed some light to the origin of ultra high energy cosmic rays (UHECRs) and high energy neutrino production.

In this project we adopt such a hadronic interpretation. Specifically, we focus on an intriguing property of hadronic plasmas, coined as hadronic supercriticality, according to which the emitting source abruptly and efficiently releases all the energy stored in protons through photon outbursts. The photon flares that are produced can be highly variable, each lasting a short period of time and thus having a direct analogy to the GRB phenomenology. While the hadronic supercriticality has been studied thoroughly in the past [1-5], here we study for the first time this phenomenon in adiabatically expanding sources. In the following sections we demonstrate that supercriticality can be achieved even if the emitting region is expanding, and show the ways it is affected by the expansion velocity. Moreover, we show that certain initial parameters of our model can successfully reproduce the prompt emission of long GRBs.

\section{Model description}

To investigate the role of hadronic supercriticalities in the expanding source scenario we adopt the standard framework of a one-zone radiation model, assuming however that the emitting region is spherically expanding, with a constant velocity $u_{\text {exp }}$. The radius of the source is increasing as $r=r_{\text {in }}+u_{\text {exp }} t$, where $r_{\text {in }}$ is the initial radius and $t$ is measured in the comoving frame of the source. This spherical region can be thought of as the shell of shocked ejecta in the internal shock scenario for GRBs. Here, $r \approx R_{\mathrm{sh}} / \Gamma$ is the comoving width of the shell, $\Gamma$ is the bulk Lorentz factor of the variable outflow producing internal shocks, and $R_{\mathrm{sh}}$ is its distance from the GRB central engine. Moreover, we assume that the emitting region contains a tangled magnetic field of strength $B$, which decreases as the source expands, i.e., $B=B_{\text {in }}\left(r_{\text {in }} / r\right)^{a}, a>0$, where $B_{\text {in }}$ is the magnetic field value that corresponds to the initial radius of the source.

Pre-accelerated relativistic protons are injected in the source with a radially dependent luminosity, $L_{\mathrm{p}}=L_{\mathrm{p}, \text { in }}\left(r / r_{\mathrm{in}}\right)^{s}$, where $L_{\mathrm{p} \text {,in }}$ is the proton injection luminosity at $r=r_{\mathrm{in}}$ and $s$ is an index that determines the rate of injection as the source is expanding. We define the instantaneous proton compactness, a dimensionless measure of the proton luminosity, as:

$$
\ell_{\mathrm{p}}=\frac{\sigma_{\mathrm{T}} L_{\mathrm{p}}}{4 \pi r m_{\mathrm{p}} c^{3}}=\ell_{\mathrm{p}, \text { in }}\left(\frac{r}{r_{\mathrm{in}}}\right)^{s-1}
$$

where $\sigma_{\mathrm{T}}$ is the Thompson electron cross section and $m_{\mathrm{p}}$ is the proton mass. Similarly, we define the instantaneous photon compactness $\ell_{\gamma}$ as:

$$
\ell_{\gamma}=\frac{\sigma_{\mathrm{T}} L_{\gamma}}{4 \pi r m_{\mathrm{e}} c^{3}}
$$


where $L_{\gamma}$ is the bolometric photon luminosity of the source. Relativistic protons interact with the magnetic field and any soft photons existing inside the source through synchrotron radiation, proton-photon (Bethe-Heitler) pair production, and proton-photon pion production processes. As a result, photons and relativistic electron-positron pairs (i.e., secondary particles) are created. Pairs contribute to the production of photons via synchrotron and Compton processes, while photons are lost from the system because of synchrotron self-absorption and photon-photon pair production. Protons, electrons, positrons and photons are the main stable species inside the source, since pions and muons decay instantaneously, neutrons do not interact with soft photons, and neutrinos escape the source freely. Protons, in general, do not cool fast and accumulate inside the source. Therefore, as their number density builds up, they can become "targets" to their own radiation, leading to the abrupt and efficient exponentiation of photons and eventually, severe proton losses.

To study the evolution of the energy distribution of stable particles in the source as a function of the increasing comoving source radius, we developed a numerical code that solves a system of coupled differential equations summarised below:

$$
u_{\exp } \frac{\partial n_{\mathrm{j}}}{\partial r}+u_{\exp } \frac{3 n_{\mathrm{j}}}{r}+\frac{n_{\mathrm{j}}}{t_{\mathrm{esc}, \mathrm{j}}}+\mathcal{L}_{j}=Q_{j}
$$

where $t_{\mathrm{esc}, \mathrm{j}}$ is the escape timescale of species $j$ from the source, $n_{\mathrm{j}}$ is the respective differential number density, and index $j=p, e, \gamma$ refers to protons, electrons/positrons and photons, respectively. Photons can escape from the source on the light crossing times, while protons and electrons remain in the source for much longer. The remaining terms in the equation describe the injection $\left(Q_{\mathrm{j}}\right)$ and loss $\left(\mathcal{L}_{\mathrm{j}}\right)$ processes of different species (see e.g. [1,6] for the physical processes included). Pre-accelerated protons are injected in the expanding source with a power-law distribution, having an injection term:

$$
Q\left(\gamma_{\mathrm{p}}, r\right)=\frac{L_{\mathrm{p}}(r)}{\left(m_{\mathrm{p}} c^{2}\right)^{2} \int_{\gamma_{\min }}^{\gamma_{\max }} \gamma_{\mathrm{p}}^{-p+1} d \gamma_{\mathrm{p}}},
$$

where $p$ is the power-law slope, while $\gamma_{\min }, \gamma_{\max }$ are the minimum and maximum proton Lorentz factors, respectively. To better study the properties of hadronic supercriticality, we assume that the injection luminosity of accelerated electrons is much lower than the proton luminosity. Hence, the source is loaded with pairs indirectly from photohadronic and photon-photon pair production processes.

\section{The supercritical behaviour in expanding systems}

Hadronic supercriticality is manifested when a feedback condition and a marginal stability criterion are simultaneously satisfied [2,7]. The former depends on the maximum energy of the proton distribution and the latter on the density of relativistic protons inside the source. The decreasing number density and adiabatic energy losses of protons in an adiabatically expanding source might hinder the development of hadronic supercriticalities. To search for the onset of supercriticality in an expanding source, we choose a small expansion velocity $\left(u_{\exp }=10^{-2.5} \mathrm{c}\right)$ and run first our numerical code with a set of initial conditions $\left(\ell_{\mathrm{p}, \text { in }}=10^{-4.25}, r_{\mathrm{in}}=10^{11} \mathrm{~cm}\right.$, $\gamma_{\mathrm{p}, \max }=10^{8}$, and $\left.B_{\mathrm{in}}=10^{4} \mathrm{G}\right)^{1}$ that drives a non-expanding system to supercriticality [5]. For

\footnotetext{
${ }^{1}$ We set $\gamma_{\min }=1$ and $p=2$ in all simulations. Here we also assume that $s=0$ and $a=0$.
} 

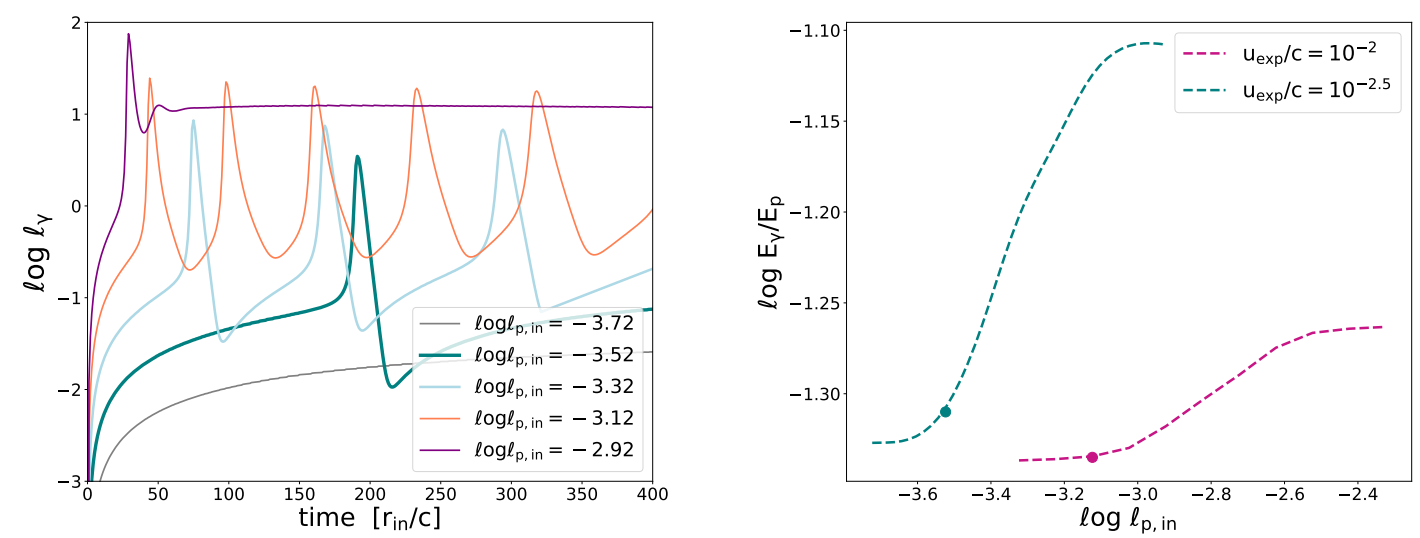

Figure 1: Left panel: Photon compactness (in $\log$ scale) as a function of time in $r_{\text {in }} / c$ units for different values of $\ell_{\mathrm{p} \text {,in }}$ and for $u_{\text {exp }}=10^{-2.5} \mathrm{c}$. Right panel: Log-log plot of the radiative efficiency, as a function of $\ell_{\mathrm{p}, \text { in }}$, computed for $t_{\text {end }}=300 r_{\text {in }} / c$, for $u_{\text {exp }}=10^{-2.5} c$ and $u_{\text {exp }}=10^{-2} c$ (green and purple lines respectively). The initial parameters are $r_{\text {in }}=10^{11} \mathrm{~cm}, \gamma_{\mathrm{p}, \max }=10^{8}$, and $B_{\text {in }}=10^{4} \mathrm{G}$, while we assume that the magnetic field and the proton injection are constant in time ( $s=0$ and $a=0$ ).

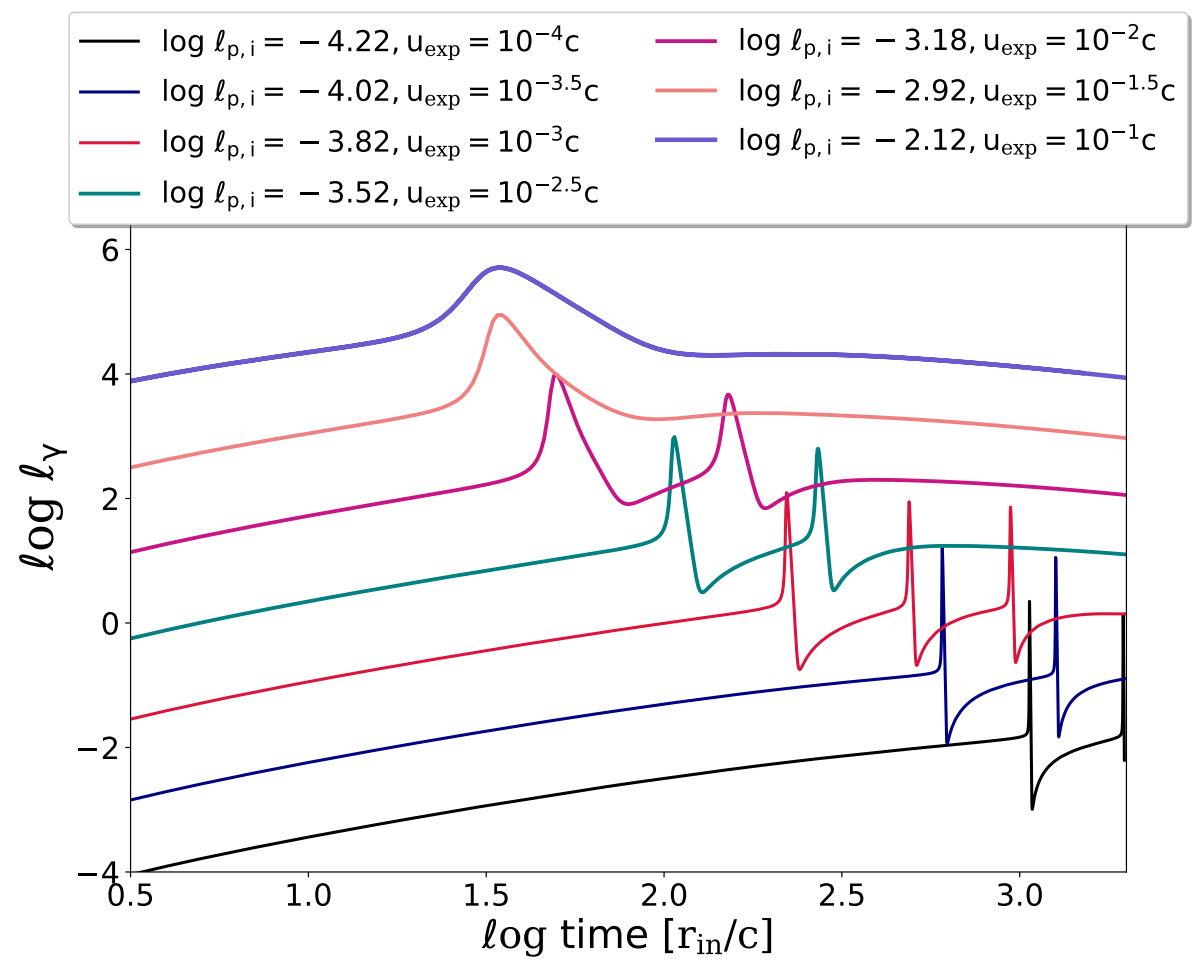

Figure 2: Photon compactness (in log scale) as a function of time for different values of $u_{\text {exp }}$ and for $\ell_{\text {p,in }}$ values for which the system firstly reenters the supercritical regime. The initial parameters are $r_{\text {in }}=10^{11} \mathrm{~cm}$, $\gamma_{\mathrm{p}, \max }=10^{8}, B_{\text {in }}=10^{4} \mathrm{G}, s=0$ and $a=0$. 
these values, however, when expansion is included, no transition to supercriticality is found. Higher proton compactnesses are needed to counterbalance the decrease in the number density due to expansion. For $\ell_{\mathrm{p} \text {,in }}=10^{-3.52}$ we recapture the non-linear behaviour (green solid line on the left panel of Fig 1. For higher values of $\ell_{\mathrm{p} \text {,in, }}$, more frequent outbursts are manifested, while the first burst progressively appears at earlier times. The internally produced photons are increased exponentially and as a result the abrupt increase of the system's radiative efficiency can be seen in the right panel of Fig 1. The green dashed curve is constructed from the parameters of our example case. The marker corresponds to the $\ell_{\mathrm{p} \text {,in }}$ that results in the first bursty lightcurve. This preliminary result shows that expansion can indeed suppress the supercritical behaviour, by dropping the proton density under its critical value and thus making the problem more energy demanding.

In order to confirm this hypothesis we rerun the code for a higher value of expansion velocity, e.g. $u_{\exp }=10^{-2} c$. For the same initial parameters the supercritical behaviour is once again lost. One needs to increase $\ell_{\mathrm{p} \text {,in }}$ even more (by a factor of 0.4 in logarithmic scale) to recapture the first burst. We compute again the system's radiative efficiency and overplot the result in the right panel of Fig. 1, in order to compare with the one that corresponds to lower expansion velocity. The growth of the efficiency curve is not so abrupt in this case, confirming the hypothesis that expansion makes the supercritical behaviour less efficient. In Fig. 2 we show the light curves we obtain for different expansion velocities. Each light curve is computed for the minimum $\ell_{\mathrm{p} \text {,in }}$ required for producing a photon burst. In this plot we also observe that the increase of the expansion velocity makes the flare last longer. Moreover, for $u_{\exp } \geq 10^{-2} c$ we no longer find multiple bursts but only a single flare with a fast rise and a slow decay.

\section{The GRB prompt emission case}

To apply the expanding supercritical model to the GRB prompt emission we seek the initial parameters that satisfy the supercritical conditions and are relevant to the GRB phenomenology. We fix $B_{\text {in }}=10^{4} \mathrm{G}$ and $r_{\text {in }}=10^{11} \mathrm{~cm}$ and search for different values of the maximum proton energy in the source, $\varepsilon_{\mathrm{p} \text {,max }}$, that will lead to instability. For these simulations, we assume that the source is expanding with $u_{\text {exp }}=10^{-2.5} c$, the magnetic field strength drops linearly as the radius increases $(a=1)$ inspired by magnetohydrodynamical models of GRB jets and the proton injection rate increases linearly with radius $(s=1)$.

In Fig. 3 we plot the photon luminosity released at the moment of the peak of the first supercritical flare, $\mathrm{L}_{\gamma, \mathrm{pk}}$, as a function of the photon energy $\varepsilon_{\mathrm{pk}}$ that corresponds to the peak of the photon spectrum (in $\varepsilon F(\varepsilon)$ units) at that moment, both measured in the observer's frame. The size of the markers indicates the ratio of the proton energy density to magnetic energy density at the same moment. The colour in the plot corresponds to different values of the maximum proton energy $\varepsilon_{\mathrm{p}, \max }$ injected in the source. The obtained photon peak luminosities fall in the range of observed GRB luminosities. However, only the lower values of maximum proton energy yield photon spectra with $\varepsilon_{\mathrm{pk}} \sim 1 \mathrm{MeV}$. For $\varepsilon_{\mathrm{p}, \max } \gtrsim 10^{17} \mathrm{eV}$, the photon spectra peak at $\varepsilon \gtrsim 10 \mathrm{MeV}$. For this reason, in the following analysis we use low proton Lorentz factors, i.e. $\gamma_{\max } \simeq 10^{4}-10^{5}$.

In order to simulate a variable central engine, we assume that a discrete number of blobs $(N)$ separated by short periods without any ejection is produced at the base of the GRB jet due to an episodic energy injection there. In particular, each blob is released after $d t=60 r_{\text {in }} / c$ from 


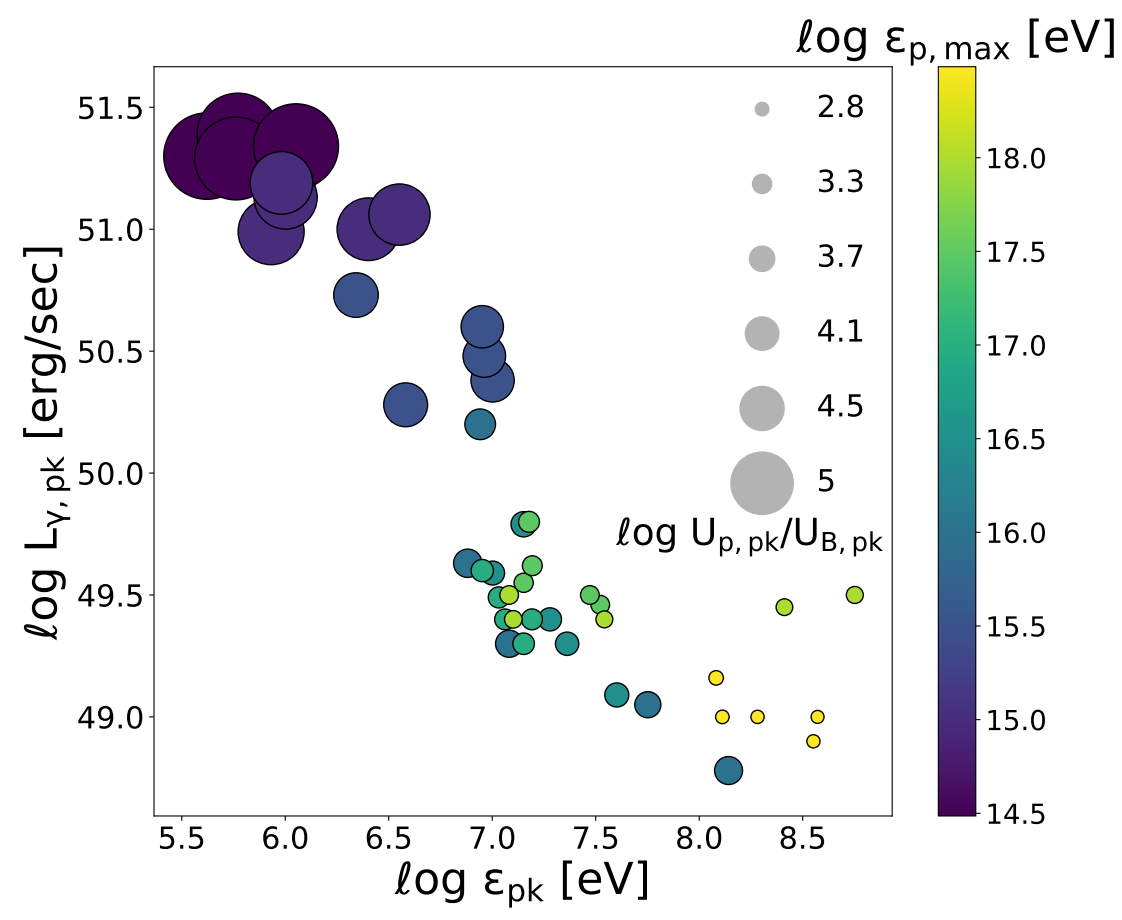

Figure 3: Scatter plot of the photon luminosity at the moment of the peak of the first flare, $\mathrm{L}_{\gamma, \mathrm{pk}}$, as a function of the photon energy that corresponds to the peak of the photon spectrum released at the same moment $\varepsilon_{\mathrm{pk}}$. The different values of maximum proton energy $\varepsilon_{\mathrm{p} \text {,max }}$ are shown in colour while the size of the source corresponds to the $U_{\mathrm{p}, \mathrm{pk}} / U_{\mathrm{B}, \mathrm{pk}}$ value at the same moment. The results are computed in the observer's frame. The fixed initial parameters are $r_{\text {in }}=10^{11} \mathrm{~cm}, B_{\text {in }}=10^{4} \mathrm{G}$ and $u_{\text {exp }}=10^{-2.5} c$ while we have assumed that $\Gamma=100$ and $\mathrm{z}=2$ as typical values for Bulk Lorentz factor and redshift of a GRB.

the previous one and the central engine emits $N=10$ blobs. For simplicity, we assume that the blobs are moving with the same bulk Lorentz factor $\Gamma$ and expand adiabatically at the same rate $\left(u_{\exp }=10^{-2.5} c\right)$.

We perform a Monte Carlo simulation and randomly select the physical parameters of each blob which ensure the onset of supercriticality. We assume that the initial magnetic field strength is following a Gaussian distribution, having a mean value at $\ell$ og $\mathrm{B}_{\text {in }}=4.3 \mathrm{G}$ and a standard deviation $\ell \operatorname{og} \sigma=0.2$. The initial proton luminosity injected is derived under the assumption that it in equipartition with the initial magnetic field energy density $U_{\mathrm{B}, \mathrm{in}}=B_{\mathrm{in}}^{2} / 8 \pi$,

$$
L_{\mathrm{p}, \mathrm{i}}=0.5 \kappa B_{\mathrm{in}}^{2} r_{\mathrm{in}}^{2} c .
$$

Here, $\kappa$ is the equipartition ratio, which we set equal to 100 . For the adopted parameters, all emitted blobs show supercritical behaviour. We compute the $\gamma$-ray light curve and the broadband photon spectrum from the superposition of blobs, without taking into account the time delay between the photons emitted by the previous and following blobs. We therefore present a physical picture of a typical GRB prompt emission in the two top panels of Fig 4. On the left plot we show the superposition of the blobs' light curves in black and in the inset plot each blob contribution in colour. 
Table 1: The energetics of the supercritical model,e.g the radiative efficiency of the model $\epsilon_{\mathrm{eff}}$ (defined as the ration of the photon energy released to the proton energy injected during the episode), the isotropic energy $E_{\mathrm{iso}}$, the photon $\varepsilon_{\mathrm{pk}}$ and neutrino $\varepsilon_{\nu \mathrm{pk}}$ energies at the peak of the total spectrum, the ratio of the photon to neutrino fluences at the peak of the spectra $\mathcal{F}_{\gamma, \mathrm{pk}} / \mathcal{F}_{\gamma, \mathrm{pk}}$ and the duration of the flaring episode $\mathrm{T}_{90}$. The peak values of the Gaussian distributions used are $B_{\mathrm{pk} \text {,in }}=10^{4.3} \mathrm{G}, L_{\mathrm{p}, \mathrm{pk} \text {,in }}=10^{42.7} \mathrm{erg} / \mathrm{sec}, \gamma_{\mathrm{max}, \mathrm{pk}}=10^{5}$ and the standard deviation $\sigma=1.58$. In this example case we use the redshift value of GRB 130505A, $z=2.27$ in order to compare the spectral results.

\begin{tabular}{lcccccc}
\hline$\Gamma$ & $\epsilon_{\mathrm{eff}} \%$ & $E_{\text {iso }}[\mathrm{erg}]$ & $\varepsilon_{\mathrm{pk}}[\mathrm{eV}]$ & $\varepsilon_{\nu \mathrm{pk}}[\mathrm{eV}]$ & $\mathcal{F}_{\gamma, \mathrm{pk}} / \mathcal{F}_{\nu, \mathrm{pk}}$ & $\mathrm{T}_{90}[\mathrm{sec}]$ \\
\hline 100 & 23.7 & $5.68 \times 10^{51}$ & $9 \times 10^{5}$ & $1.6 \times 10^{13}$ & 7.9 & 60 \\
\hline
\end{tabular}

The result is a multi-burst pulse that lasts approximately a minute and has luminosity comparable to observed GRB light curves. On the right panel of the same figure we show the superposition of the photon fluence with the dashed black line and each blob's contribution in colour. The energy that corresponds to the peak of the photon total fluence, has got a typical value observed in a GRB prompt emission. We also provide the all-flavour neutrino flux expected under the assumptions we have made and compare our result with the expected fluence of GRB 130505A (red dotted line) studied in [8]. It seems that the neutrino energy at the peak of the spectrum is lower by a factor of two in logarithmic scale compared to the example case of the standard internal shock neutrino model. Moreover, the numerically calculated neutrino fluence is one order of magnitude lower than that of the example case. In Table 1 we summarise the results of our supercritical GRB model.

We also calculate the isotropic energy released in photons emitted by integrating the total fluence $\mathcal{F}_{\gamma}(\varepsilon)$ (black dashed line of Fig 3) over the observed photon energies:

$$
E_{\text {iso }}=4 \pi d_{\mathrm{L}}^{2} \int_{\varepsilon_{\text {min,obs }}}^{\varepsilon_{\max , \text { obs }}} \mathcal{F}_{\gamma}(\varepsilon) d \varepsilon
$$

where $d_{\mathrm{L}}$ is the luminosity distance of the central engine and $\varepsilon_{\min , o b s}, \varepsilon_{\max , \text { obs }}$ the minimum and maximum photon energies of the observed spectrum respectively.

\section{Conclusions}

In this project we show that hadronic supercriticality exists even in expanding sources. As the expansion velocity is increased, the manifestation of supercriticality becomes more energy demanding while the spectral behaviour of the system changes from a multi-burst to a broader single burst one. We work on a model of randomly emitted supercritical blobs that are expanding with the same velocity and do not collide with each other in order to reproduce a typical long GRB. The initial paramerers used fall in the case of GRB prompt emission as well as lead the expanding blobs to supercriticality. We choose the lower values of maximum proton energy (i.e. $\gamma_{\max } \simeq 10^{4}-10^{5}$ ), because such values yield photon spectra that peak at $1 \mathrm{MeV}$. The computed isotropic energy released as well as the produced spectra are in good agreement with the observations. Moreover, the constructed all-flavour neutrino spectrum peaks at lower neutrino energies compared to a standard internal shock model [8]. We infer that this is the result of the low values of $\gamma_{\max }$ used in the present example case. As well as that the peak neutrino fluence is lower in our case. Overall, our model can rather satisfactorily mimic a GRB prompt emission. 

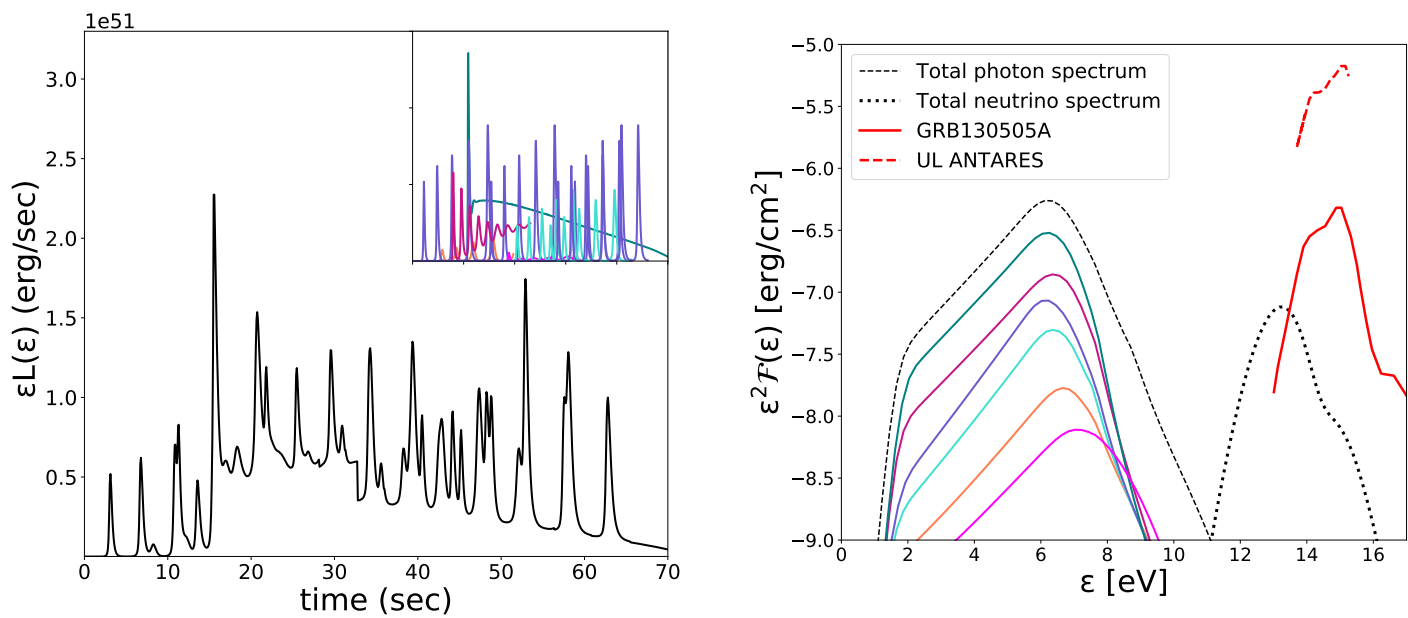

Figure 4: Left panel: The superposition of supercritical lightcurves (black line) and the contribution of each blob in the inset plot. Right panel: The superposition of the photon (dashed black line) and neutrino (dotted black line) spectra. In colour we show the photon spectrum of each blob. The red dotted and dashed-dotted lines correspond to the expected fluence and ANTARES 90 per cent C.L. upper limits on the selected GRB [8] respectively. We have rescaled the latter fluences for comparison reasons. In this example we assume the redshift value of the example GRB, $z=2.27$ and as Bulk Lorentz factor $\Gamma=100$.

\section{Acknowledgments}

This research is co-financed by Greece and the European Union (European Social Fund-ESF) through the Operational Programme «Human Resources Development, Education and Lifelong Learning» in the context of the project "Strengthening Human Resources Research Potential via Doctorate Research" (MIS-5000432), implemented by the State Scholarships Foundation (IKY).

\section{References}

[1] A. Mastichiadis, R.J. Protheroe and J.G. Kirk, Spectral and temporal signatures of ultrarelativistic protons in compact sources - i. effects of bethe-heitler pair production, A\&A 433 (2005) 765.

[2] M. Petropoulou and A. Mastichiadis, Temporal signatures of leptohadronic feedback mechanisms in compact sources, MNRAS 421 (2012) 2325 [1201.2091].

[3] M. Petropoulou, S. Dimitrakoudis, A. Mastichiadis and D. Giannios, Hadronic supercriticality as a trigger for $\gamma$-ray burst emission, MNRAS 444 (2014) 2186 [1407.2915].

[4] M. Petropoulou and A. Mastichiadis, Patterns of variability in supercritical hadronic systems, MNRAS 477 (2018) 2917 [1804.00188].

[5] A. Mastichiadis, I. Florou, E. Kefala, S.S. Boula and M. Petropoulou, A roadmap to hadronic supercriticalities: a comprehensive study of the parameter space for high-energy astrophysical sources, MNRAS 495 (2020) 2458-2474.

[6] N.S. Kardashev, Nonstationarity of Spectra of Young Sources of Nonthermal Radio Emission, azh 39 (1962) 393.

[7] J.G. Kirk and A. Mastichiadis, X-ray flares from runaway pair production in active galactic nuclei, NAT 360 (1992) 135.

[8] A. Albert, M. André, M. Anghinolfi, G. Anton, M. Ardid, J.-J. Aubert et al., Search for high-energy neutrinos from bright grbs with antares, MNRAS 469 (2017) 906-915. 